\title{
2. Bunzl on Sorensen's Thought Experiments
}

\section{MARTIN BUNZL Rutgers University}

Roy Sorensen's book, Thought Experiments' ${ }^{1}$, stands in a crowd of recent work that represents a new philosophical industry focusing on thought experiments. ? Some of this literature is polemical, while a lot of it is argued very close to the ground. In his book, which is engagingly written and wide ranging in scope, Sorensen aspires to give a treatment of the whole area - from origins, to logical structure, to use.

More than anything, $I$ think Sorensen's contribution is that his book offers a wealth of material on the cognitive function of thought experiments and on their use and misuse relative to this function. He argues that thought experiments do their cognitive work in a variety of different ways: ... by selective reminder, transformation, deletion, rearrangement, and cleansing ( 1 take these terms from Sorensen) of the intellectual suppositions that we bring to our thinking.

But, notwithstanding these contributions and Sorensen's broad ambitions, I want to concentrate on just one area of thought experiments: the issue of their epistemic contribution. (I do so, because I take this to be the central area of challenge for anyone who wants to make the case of thought experiments.) There is no doubt that thought experiments make non-epistemic contributions. And of course, one can count the cognitive role of thought experiments that Sorensen champions as epistemic. But I am narrow minded: I think "real" experiments make epistemic contributions to our theories - in the form of constituting an independent source of data about the world. If thought experiments are really experiments don't they have to do the same thing?

Sorensen wants to defend thought experiments as experiments. And he has an answer to narrow minded types like me. His argument is that types like me have an overly narrow view of "real" experiments. Sorensen thinks that experiments do all sorts of important things .... only one of which is providing fresh information about the world. And, providing fresh information about the world is only one way in which the persuade us. They also persuade us:

by methods associated with armchair inquiry: ... [by what I called, above, the cognitive role of thought experiments]. Once we see that these factors were there all along, we can stop viewing thought experiment as introducing a new mode of persuasion. Thought experimentation only reveals the hidden persuaders involved in ordinary experiments by taking away the most obvious persuader (251).

The talk of 'persuasion' is noteworthy here - one disagreement I have with Sorensen is about where we draw the boundaries of just where experiment ends 
and the use to which experiment is put begins. For me the persuasiveness of experiments in the context of justifying our theoretical commitments is not part of the experiments themselves but a use to which they are put. The difference is important because, if I am right, it is not so obvious that the features of thought experiments that Sorensen values fall under the umbrella of experiments as opposed to the use to which experiments can be put. Be that as it may, even if Sorensen is right in thinking of thought experiments as being like "real" experiments in lots of important ways and just different in one respect, what gets left unaddressed in this formulation is the question of to what degree this one respect is the crucial one. Furthermore, and more importantly, I don't see how the cognitive virtues of "real" experiments can be unbundled from experiment as a source of fresh information. For absent fresh information, we deprive ourselves of the most obvious source for making judgments as to the reliability of experiments which, in turn, deprives us of the most obvious basis for deciding between the claims of competing experiments which will include competing outcomes qua their cognitive functions.

Sorensen has an answer to this as well - but an answer that I find implausible - it is an evolutionary ploy. Under the influence of Mach, he argues that our minds have developed in such a way as to select out for the reliability of some of our modal intuitions. ${ }^{3}$ Sorensen wants to claim that:

\begin{abstract}
Biology gives us further clues when we concentrate on the kinds of problems our modal intuitions were designed to solve. The hunter-gatherer needed to track objects in space and time; to distinguish friend from foe; and to recognize predator and prey, shelter and foodstuffs. Moreover, he needed to manipulate objects and predict behavior. Thus we have a rich stock of intuitions about space and time and biological and social relationships and a great aptitude for belief-desire psychology and causal reasoning. Humans are also endowed with excellent visual judgment and mastery of language. Hence, thought experiments that exploit these intuitions and abilities, whether directly or through resourceful indirection, are apt to fare well (253).
\end{abstract}

I am generally skeptical about philosophical appeals to evolutionary arguments .... they are just too easy! But in this case I think the thesis is testable and I think it fails the test: if you look at philosophical thought experiments, some are distinguished by the straightforwardness and robustness with which their conclusions follow. On the other hand, there are others that seem to have the capacity to spin out of control quite easily. Compare Gettier type cases with personal identity transfer cases. Neither of these appeal to the features Sorensen cites as being selected for, yet they are strikingly different. As Bernard Williams argued, outcomes in transfer cases are easily perturbated depending on the mode of presentation. ${ }^{4}$ And in general, when it comes to personal identity cases, individual intuitions seem to be notoriously heterogeneous. And yet none of this seems characteristic of Gettier type cases. Why not? If the answer is not evolution an obvious alternative grounding, experience, will fare no better. I think the right answer is this: the Gettier case involves inference while the Williams cases involve imagination. 
The notion of dividing thought experiments into those that are simply inferential and those that involve the imagination (or intuition) provides a taxonomy with which to differentiate positions in the recent literature of thought experiments ... although as Sorensen points out, the distinction can be found in Hempel.s I think this is a sound distinction and much ground-clearing can be done by appealing to it. Though I won't argue for it in detail here, I think that the only reliable thought experiments are simply inferential in nature. When they work, what such experiments achieve is a demonstration of an untoward consequence of a generalization and a set of background assumptions.

Even though Sorensen thinks that thought experiments do their work by way of our (evolutionarily refined) intuitions, he also thinks we can capture the structure of all experiments as what he terms either necessity refuters or possibility refuters. (I only take up the former here, though parallel remarks apply to the latter.) The idea is this: think of the modal implications of a theory. Combine these with conditions to generate a counterfactual that includes what Sorensen calls a "weird consequence"(136). That weirdness can then be explained by appealing to the impossibility of the consequent of the counterfactual and hence to a finding about the antecedent of the counterfactual. From there we can reject the modal implication of the theory (assuming we accept the thought experiment) and hence (unless we reject the inference from the theory to the modal implication) we can reject the theory itself. Presumably our intuitions come into play in the counterfactual judgments (see 35-136).

What should we make of this analysis? I should say at the outset that I am puzzled about how to reconcile it with Sorensen's commitment to analyzing thought experiments as realizing the kinds of cognitive functions I summarized at the outset. Why should such functions require all of this logical machinery?

More generally, I am puzzled by the appeal to the modal, particularly when you remember that this is an analysis that is meant to apply to "real" experiments as well as thought experiments. Surely all those mundane and painstaking studies that are the bread and butter of experimental science don't do anything this logically extravagant. At most they confirm or falsify simple predictions of theories not modal claims - either of necessity or possibility. Nor are the modalities in such extravagant evidence when it comes to thought experiments either. Nor do 1 think we need to appeal to our intuitions. Instead, things are much simpler. We begin with a universal claim (be it part of a scientific theory or part of a philosophical theory). To this we add background assumptions and plug in initial conditions. Of course the result cannot directly confirm a theory in this way. But to the extent that the theory applies everywhere, of course the result can undermine the theory by generating a consequence that is unacceptable - where unacceptable can range from inconsistency to merely placing well confirmed other background assumptions in question.

And, keeping in mind my epistemic narrow mindedness, that is all I think that good thought experiments do in both science and philosophy. I don't dispute 
that there are other uses for thought experiments if one is more broad-minded: when two theories compete, the thought experiment that undermines one will thereby indirectly lend confirmation to the other. And, independent of these functions, thought experiments may play a role in demonstrating the range of a theory and in providing a framework within which to reconceive of a theory's range. But here a cautionary note is appropriate: I think thought experiments play a much smaller role in science than many philosophers seem to think. Take Einstein - Sorensen calls him "the grandmaster of Gedankenexperiment"'(209). Yet look at Einstein's thought experiments that are frequently cited - with the exception of EPR they appear in popular treatments of theories, written for general audiences, or in the introductory paragraphs of essays before the mathematics kicks in to do the real work. Take the opening paragraph of the 1905 paper, "On the Electrodynamics of Moving Bodies". The thought experiment goes like this: we are asked to consider the observable phenomena in the electrodynamic interaction between a magnet and a conductor - it is only the relative motion that counts. But this is hardly intended as a surprising claim. It is simply an illustration of the fact that:

It is well known that Maxwell's electrodynamics - as usually understood at present - when applied to moving bodies, leads to asymmetries that do not seem to attach to the phenomena. ${ }^{6}$

On the other hand, some of the most well known of Einstein's thought experiments - the elevator or the chest and the railway carriage - have their origin in his popular writings and don't even appear in scientific papers.?

But what about the use of thought experiments to provide us with a locale within which we can clarify our own rules for the application of concepts? Here too I want to sound a cautionary note. Of course you can reject this project on Wittgensteinian grounds, but a much more modest basis on which to do is simply that such an exercise is less and less interesting the closer the locale is to home. And yet the further we get from home the less reliable our intuitions except insofar as we covertly rely on general principles to guide us.

Take Twin Earth cases. It is easy to forget that Putnam's argument that extension is not determined by psychological state won't work unless you assume continuity of reference before and after the discovery of the molecular structure of water - which follows from the argument from natural kinds. Burgean versions only work by assuming that we can read back from that-clauses to intentional mental states and that we can give a satisfactory gloss on common practice when it comes to the attribution of propositional attitudes. These are not claims that fall out of running thought experiments but rather claims we bring to the thought experiments themselves. But not all Twin Earth cases have these features. In Putnam's treatment of non-natural kind terms (the case of the living "pencils") there is no general claim that forces the counterfactual judgment he thinks we would make and thus (not surprisingly) 1 think the counterfactual judgment is much less robust than his conclusions involving natural kind terms. ${ }^{*}$

I end with a reservation about my own comments that is a version of (Hempel's) Theoretician's Dilemma: one role for thought experiments in 
philosophy might appear to be an argument by analogy. In Thomson's violin case the thought experiment is meant to be like the rape case in morally relevant respects. The violin case is obviously subsumable under a general analysis of rights and consent. (In fact, I would argue that the reason we choose the case is because it straightforwardly instantiates these general principles.) The function of the thought experiment is thus to allow us to see how to redescribe the rape case thereby making it subsumable under the same moral principles. Of course once we have achieved that goal, it is easy to excise the thought experiment as unnecessary baggage. But of course that does not render thought experiments as dispensable - any more than theoretical entities are dispensable even if we can Ramsify them away.

\section{Notes}

'R. Sorensen, Thought Experiments (New York: Oxford University Press, 1992). (All page citations in the text are to this work.)

${ }^{2}$ See, J.Brown, The Laboratory of the Mind (London: Routledge, 1991) T. Horowitz and G. Massey, Thought Experiments in Science and Philosophy (Savage, Maryland: Rowman \& Littlefield, 1991), and K. Wilkes, Real People (Oxford: Clarendon, 1988).

${ }^{3}$ As Mach would have it: "Everything which we observe in nature imprints itself uncomprehended and unanalyzed in our percepts and ideas, which, then, in their turn, mimic the processes of nature in their most general and most striking features." E. Mach. The Science of Mechanics (LaSalle, III.: Open Court, 1942), page 36.

"See, B. Williams, "The Self and the Future," Philosophical Review 79 (1970):161-180.

${ }^{5}$ Carl Hempel, Aspects of Scientific Explanation (New York: Free Press, 1965), pp.164-165.

'Analen Der Physik 17 (1905):891.

${ }^{7}$ See A. Einstein and L. Infeld. The Evolution of Physics (New York: Simon and Schuster, 1938), pp.226-239, and A. Einstein, Relativity (New York: Henry Holt and Co., 1921), pp.69-73 and pp.78-83. I don't want to overgeneralize here: Einstein's less frequently discussed thought experiments need to be taken on a case by case basis. In "The Foundation of General Relativity" (1916), a thought experiment is raised simply to make an epistemological point about the status of theories about states of affairs that are not observationally distinct. On the other hand, in the 1909 papers on radiation, they constitute the central case in the representation of a hypothetical used to overthrow features of the then prevailing theory of radiation. (I have benefited frum Norton's discussion of Einstein in the Horowitz and Massey volume, notwithstanding my disagreement with his analysis of them. See J. Norton, "Thought Experiments in Einstein's Work," in T. Horowitz and G. Massey, op. cit., pp.129-148.

"See H. Putnam, "The Meaning of Meaning," Mind. Language and Reality (Cambridge: Cambridge University Press, 1975), pp. 215-271 and T.Burge, "Individualism and the Mental," Midwest Studies in Philosophy, Vol. 4 (Minneapolis: University of Minnesota Press, 1979), pp.73-121.

MARTIN BUNZL 